Available online on 15.11.2016 at http://jddtonline.info
(c) 2016, publisher and licensee JDDT, This is an Open Access article which permits unrestricted
noncommercial use, provided the original work is properly cited

Research Article

\title{
NEW SLANT ON HYPERLIPIDEMIA: PREVENTION BY VITAMIN B-3 AND COMMON FRUIT ZIZIPHUS JUJUBA
}

\author{
Khalid Niaz, Ajaz Fatima, M. Ashraf Memon, Shahina Hakro, Shah Murad \\ Islamabad Medical and Dental College, Islamabad-Pakistan (Affiliated with Shaheed Zulfiqar Ali Bhutto Medical University, Islamabad)
}

\begin{abstract}
Coronary artery disease (CAD) is complication of primary or secondary hyperlipidemia. Etiological factors for hyperlipidemia include inherited genetic defects in low density lipoproteins (LDLs), persons accustomed to sit for prolong time, cigarette smoking, diabetes mellitus, and hypertension. This research work's aim was to compare hypolipidemic features of an allopathic agent vitamin B-3 and another medicinal plant Z. Jujuba. Study was conducted from January 2016 to June 2016 at Jinnah Hospital Lahore, Pakistan. 60 male and female hyperlipidemic patients age range from 20 to 70 years were selected from the hospital. Written and explained consent was taken from all patients. They were divided in two groups. Group-I was advised to take two grams vitamin B-3 in divided doses for the period of two months. Group-II was advised to take 500 grams of fruit Z. Jujuba daily for 60 days per their convenience. Their initial LDL and HDL cholesterol was determined by Friedwald's formula. After 60 days therapy, their post treatment lipid profile was measured and mean values with \pm SD were analyzed biostatistically by using SPSS version 2; 2010 . Group-I which was on vitamin B-3 their LDL cholesterol decreased significantly and HDL cholesterol was increased significantly. In group-II patients LDL cholesterol was decreased significantly but HDL increase was not significant with p-value of $>0.05$. It was concluded from the research study that vitamin B-3 is potent hypolipidemic agent as compared to medicinal plant Z. Jujuba.
\end{abstract}

Article Info

Received 13 Oct 2016; Review Completed 04 Nov 2016; Accepted 07 Nov 2016, Available online 15 Nov 2016

Article URI: http://jddtonline.info/index.php/jddt/article/view/1342

Cite this article as:

Niaz K, Fatima A, Memon MA, Hakro S, Murad S, New slant on hyperlipidemia: prevention by vitamin B-3 and common fruit Ziziphus jujuba, Journal of Drug Delivery \& Therapeutics. 2016; 6(6):22-24 DOI: http://dx.doi.org/10.22270/jddt.v6i6.1342

*Corresponding author:

Prof. Dr. Shah Murad Mastoi, Professor \& HOD of Pharmacology, Islamabad Medical and Dental College, Islamabad-Pakistan (Affiliated with Shaheed Zulfiqar Ali Bhutto Medical University, Islamabad)

Email: shahhmurad65@gmail.com, PHONE: 0092512807201, 009203142243415

\section{INTRODUCTION:}

Antihyperlipidemic agents promote reduction of lipid levels in the plasma ${ }^{1}$. Some antihyperlipidemic agents aim to lower the levels of low-density lipoprotein (LDL) cholesterol, some reduce triglyceride levels, and some help raise the high-density lipoprotein (HDL) cholesterol. Reducing the LDL cholesterol, they can cure both the primary and secondary hyperlipidemia ${ }^{2-7}$. Vitamin B-3 or Niacin, Statins, Fibric acids, Bile Acid Binding Resins are main drug groups used in allopathy ${ }^{8-}$ ${ }^{9}$. Basic problem with use of vitamin B-3 is its dose ie; 2 grams daily to lower cholesterol in plasma. In this dose it causes flushing and urticaria ${ }^{10}$. Statins and Fibrates cause rhabdomyolysis and muscular pain ${ }^{11-14}$. If used for long time, bile acid binding resins cause withdrawal by patient due to its metallic taste ${ }^{15}$. Alternative hypolipidemic therapy include medicinal herbs like $\mathrm{Z}$ Jujuba which reduce LDL cholesterol, triglycerides, total cholesterol and somewhat incraese HDL cholesterol $^{16}$. Active ingredients of $\mathrm{Z}$ Jujuba include colubrinic acid, zizyberenalic acid, alphitolic acid, alphitolic acid, betulinic acid, betulonic acid, maslinic acid, oleanolic acid, oleanonic acid. These Zizyphus jujuba compounds were examined for their antioxident 
characteristics and proved mild to moderate free radicals scavenger compunds ${ }^{17-20}$. Some researchers explained that, a number of compounds are present in Z. jujuba, including saponins, jujubosides and triterpenoic acids which are antioxidant compounds in nature ${ }^{21}$. In some meta analysis studies it was proved that major hypolipidemic mechanism of $\mathrm{Z}$ Jujuba is that compounds present in the plant bound with bile acids in intestine and excrete them out, thus interrupting enterohepatic circulation. This will cause hepatocytes to synthesize bile acids instead of cholesterol ${ }^{22}$.

\section{MATERIAL/SUBJECTS/METHOD:}

This work was conducted at Jinnah Hospital Lahore Pakistan from January 2016 to June 2016. 60 male/female hyperlipidemic patients were enrolled for study. Consent was taken from all participents. Research work permission were approved by ethics committee of the hospital. Both male and female patients suffering from primary or secondary hyperlipidemia were selected. The age limit for patients was 20 to 70 years. Alcoholic addictive patients, cigarette smokers, habitual to enjoy sedentary life, with impaired liver or renal functions were excluded from the research study. Baseline Lipid Profile was determined in Biochemistry lab of the Hospital. Serum cholesterol was estimated by enzymatic method. Serum HDL-cholesterol was determined by using kit Cat No: 303210040 by Elli Tech Diagnostic, France. Chylomicrons, low density lipoprotein and very low density lipoprotein are specially precipitated with phosphotungstic acid and magnesium ions can then be removed by centrifugation, while high density lipoproteins remain in the supernatant. Cholesterol included in this phase is measured by an enzymatic method. LDL-cholesterol was calculated according to Friedwald formula (described by laralaoi $\mathrm{J}$ et $\mathrm{al}^{21}$ ) i.e.; $\mathrm{LDL}=\mathrm{TC}-(\mathrm{TG} / 5+$ HDL-C). Patients were divided in two groups, 3o patients in each group. Group-I was on Tab. Niacin 2 grams daily in three divided doses. Group-II was on Jujube 500 grams daily in three divided times to eat. They were advised to take this fruit for two months. Mean values \pm SD were taken for statistical analysis using SPSS version 26 (2015). Paired' $t$ ' test was applied to get significance changes in parameters before and after treatment. P-value $>0.05$ was considered as nonsignificant change, p-value $<0.01$ was considered as significant and p-value $<0.001$ was considered as highly significant change in the tested parameter.

\section{RESULTS:}

When results were compiled after statistical analysis it was observed that with 60 days therapy by vitamin B-3 LDL-cholesterol in 27 hyperlipidemic patients reduced $29.2 \mathrm{mg} / \mathrm{dl}$ and HDL increase in this group was 7.3 $\mathrm{mg} / \mathrm{dl}$. Three patients withdrew from the study in this group due to flushing in dependant parts of the body like hanging lower earlobes, tip of nose, testicles, fingers and palm of upper and lower limbs, female breast nipples. Basically it is not a adverse effect of vitamin B-3, rather it is beneficial effect of the vitamin. Because this flushing effect is produced by release of prostaglandin
D-2 from endothelium of blood vessels, which causes vasodilatory effect on dependent parts of human body. Other participants were counseled carefully regarding vitamin B-3 dose titration, ie starting from low dose to therapeutic dose. In group-2 $(n=30)$ two months therapy by Z. Jujuba reduced LDL-cholesterol from $198.82 \pm 2.17 \mathrm{mg} / \mathrm{dl}$ to $190.91 \pm 1.73 \mathrm{mg} / \mathrm{dl}$, which is 7.9 $\mathrm{mg} / \mathrm{dl}$ change in the parameter. HDL-cholesterol in this group increased from $38.6 \pm 2.19 \mathrm{mg} / \mathrm{dl}$ to $41.9 \pm 2.97$ $\mathrm{mg} / \mathrm{dl}$, which is $3.3 \mathrm{mg} / \mathrm{dl}$ change in the parameter. Biostatistically it is non-significant change in the tested parameter of lipid profile. When analyzed in percentage change it was seen that vitamin B-3 reduced LDL cholesterol $13.9 \%$ in 27 hyperlipidemic male and female patients in 60 days therapy. HDL cholesterol in this group increased was $4.0 \%$. In Z. Jujuba group LDL cholesterol reduction in 30 male and female hyperlipidemic patient's was $16.2 \%$ and HDL cholesterol increase was $7.9 \%$.

\section{DISCUSSION:}

Myocardial infarction, congestive cardiac failure, cardiac arrhythmias, and hypertension are complications of coronary artery disease, which are initiated by formations of atherosclerotic plaques due to primary or secondary hyperlipidemia. We can prevent all these complications simply by lowering plasma lipids. LDL is main cause of oxidation which form foamy cells ie atheroma, which is sticked within inner walls of coronary arteries causing CAD. Simply by reducing bad cholesterol ie; LDL cholesterol and increasing good cholesterol ie; HDL cholesterol, we can prevent CAD. In our research study 2 grams of vitamin B-3 reduced LDL cholesterol in 60 days significantly. It also increased HDL cholesterol significantly. Palve T et $\mathrm{al}^{23}$ and Salouit $\mathrm{E}$ et $\mathrm{al}^{24}$ proved same results when they used 2 grams of vitamin B-3 in 66 hyperlipidemic patients, but Mao Ki et $\mathrm{al}^{25}$ observed lesser effects of Niacin on HDL cholesterol, i.e. only $4.4 \%$ increase in HDL cholesterol. Grams ET et $\mathrm{al}^{26}$ explained different mechanisms of hypolipidemic response of vitamin B-3 on persons with different genetic code. One of the favorable mechanism for patients with CAD they described is fibrinolytic activity of vitamin B-3. In our results Z. Jujube fruit decreased LDL cholesterol is 7.9 $\mathrm{mg} / \mathrm{dl}$, which is significant change in the parameter. HDL cholesterol is not increased significantly in our results with $\mathrm{p}$-value of $>0.05$. Yulkat $\mathrm{Y}$ et $\mathrm{al}^{27}$ and Saloiu TL et $\mathrm{al}^{28}$ observed same reason of Jujube on LDL and HDL-cholesterol, which augment our results. Mekawali PO et $\mathrm{al}^{29}$ observed more effects of Jujube as we observed in low density lipoprotein cholesterol. John $\mathrm{DB}$ et $\mathrm{al}^{30}$ proved that if given in large amount $\mathrm{Z}$ Jujuba can lower LDL cholesterol but HDL cholesterol is not increased remarkably. They also mentioned mode of action of Jujuba that it inhibit enterohepatic circulation of bile, causing synthesis of bile acids in liver, instead of cholesterol synthesis. Many researchers do not agree to replace allopathy related drug regimens for hyperlipidemia by medicinal plants due to wide variety of pharmacological actions of medicinal herbs. They do emphasize on more research work on medicinal herbs, 
so that patient may not suffer from synergetic effects of foods and these herbs. We do agree with this new slant on medicinal herbs. Suleman $\mathrm{T}$ et $\mathrm{al}^{31}$ described hazards of food-herb interaction, when patients or even physicians/cardiologists are not so aware about combined effects of these foods and herbal medicines.

\section{CONCLUSION:}

It was concluded at end of the research work that Vitamin B-3 has remarkable beneficial effects on LDLcholesterol reduction and HDL-cholesterol enhancing effect when compared with Z. Jujuba in hyperlipidemic patients.

\section{REFERENCES:}

1. Grundy SM, Wilhelmsen L, Rose G, Campbell RWF, Assmann G. Coronary heart disease in high-risk populations: lessons from Finland. Ethop Heart J 2015;17(3):1290-9.

2. Holmes CL, Schulzer M, Mancini GB. Hypolipidemic drugs for CAD. Lipid Res 2014;13(7):233-9.

3. Foulaw JU, Miller KW, Small DM. Surface-to-core and interparticle equilibrium distributions of triglyceride-rich lipoprotein lipids. J Biol Chem 2016; 258:13772-84.

4. Krauss RM. Atherogenicity of triglyceride-rich lipoproteins. Af J Cardiol 2015;81:13-7.

5. Thompson GR. Angiographic evidence for the role of triglyceride-rich lipoproteins in progression of coronary artery disease. Afr Heart J 2014;19(2):34-40.

6. Parkal CS, Gordon DJ. Cholesterol lowering agents are true drugs for CAD. Lipid Res 2015;12(6):119-22

7. Jatendaar TL, Anderson KM, Castelli WP, Levy D. Cholesterol and mortality: in Sudan. Bio Res 2014;30(9):1212-7.

8. Gaw CL, Law MR, Thompson SG, Wald NJ. Assessing possible hazards of reducing serum cholesterol. Bioch $\mathbf{J}$ 2015;308:373-8.

9. Lokavi JK, McGill HC Jr, McMahan CA. Pathobiological Determinants of Atherosclerosis and its cure. J Physiol 2016;13(9):177-9.

10. McGill HC Jr, McMahan CA, Zieske AW, Sloop GD, Walcott JV. Vitamin B-3 (Niacin) is useful but? Arterioscler Thromb Vasc Biol 2014; 20:1998-2004.

11. Takeichi S, Yukawa N, Nakajima Y, Osawa M, Saito T, Seto Y, Nakano T, Saniabadi AR, Adachi M, Wang T, Nakajima K. Association of plasma triglyceride-rich lipoprotein remnants with coronary atherosclerosis in cases of sudden cardiac death. Cl Lipid Res 2013; 19(5):333-9.

12. Polasa TR, Grundy SM. Hypertriglyceridemia, atherogenic dyslipidemia and their treatment options. J cl Res 2015;10(5):222-8.
13. Saew TR, Law MR. Lowering heart disease risk with cholesterol reduction. J cl Cardiol 2014;19:118-20.

14. Jalui J, Steiner G, Schwartz L, Shumak S, Poapst M. The association of increased levels of intermediate-density lipoproteins with smoking and with coronary artery disease. J Physiol AFR 2013;18(7):111-8.

15. Lowaree T, Kuchinskiene Z, Carlson LA. Statins and Fibrates: are these safe hypolipidemic medicines? J Cardiol 2014;12(7):345-8

16. KY Ruha, NJ Ramaj, Pk Lawal, UY Oraal. Herbs as Hypolipidemic agents for CAD cure. Lipid Res J 2015;34(4):1083-96.

17. McGill HC Jr, McMahan CA, Malcom GT, Oalmann MC, Strong JP. Effects of herbal saponins on lipids. Arterioscler Thromb Vasc Biol 2015;17:95-106.

18. Qaluiy KI, Weisgraber KH, Innerarity $\mathrm{TL}$, Rall SC Jr, Mahley RW. Herbal medicine for plasma lipids. Pharma Res 2013;23(8):222-9.

19. Lopara T, Koren E, Corder C, Mueller G, Centurion H, Hallum G. Active ingredients of $Z$ Jujuba. Pharma plant Res 2012;19(3):289-94.

20. Palsy TR, Grundy SM. Cholesterol-lowering trials by plants. J pharma 2013;18(6):712-7.

21. Laralaoi J, Karpe F, Boquist S, Tang R, Bond GM. Ziziphus jujuba for lipid therapy. J pharmacog 2013;18(2):100-2.

22. Moki LT, Havel RJ. Medicinal herbs with good pharmacological effects. J Ph Res 2014;10(1):16-20.

23. Palve TI, Torela CK, Breslow JL. Vitamin B-3 for hyperlipidemia can prevent CAD. J Pharmacol 2014;12(6):213-9.

24. Salouit E, Sacks FM, Alaupovic P, Moye LA, Cole TG, Sussex B, Stampfer MJ, Pfeffer MA, Braunwald E. Niacin or Vitamin B-3 is not only vitamin: it is cure too for hyperlipidemia. Circulation 2012;102:1886-92.

25. Mao Ki , ordestgaard BG, Lewis B. HDL cholesterol for hyperlipidemia: cause and treatment. Atherosclerosis 2014;87:39-46.

26. Grams ET, Law MR, Wald NJ, Thompson SG. Mode of action of vitamin B-3 for hypolipidemic actions. Cl Nut. 2013; 308:367-72.

27. Yulkat Y, Tatami R, Mabuchi H, Ueda K, Ueda R. Medicinal herb for Hyperlipidemia treatment. J Pharmacog 2013;12(2):116-9

28. Saloiu TL, Law MR, Wald NJ, Wu T, Hackshaw A, Bailey A. Use of Ziziphus Jujuba for LDL and HDL cholesterol. Res J Lipids 2016;12(3):177-9.

29. Mekawali PO, Mahley RW, Weisgraber KH, Innerarity TL, Rall SC Jr. Medicinal herbs for treatment of high plasma LDL particles. J Pharma Ther 2014; 265:78-83.

30. John DB, Toor M, Katchalsky A, Agmon J, Allalouf D. Z Jujuba inhibit enterohepatic circulation.J Pharma Res 2016; 22:265-7.

31. Suleman T, Fatima H, Muraka B, Farhad S. Herbs and fatty foods may cause severe adverse effects in treating hyperlipidemia. Pharma J Ther 2014; 14(4):266-9. 